\title{
Microscopy in the 64-bit Age
}

\author{
J. Wilbrink and R. T. Harmon
}

Gatan Inc., 5794 W. Las Positas Blvd., Pleasanton, CA 94588

Most of the software applications used by microscopists to operate their equipment are currently running on computers with a 32-bit operating system, most commonly Microsoft Windows. This constrains the addressable memory space to be $4 \mathrm{~GB}$ and in practice this number is reduced to $2 \mathrm{~GB}$ or less on a Windows OS. This limits the size of files that can be manipulated in memory and gives rise to laborious mapping of file regions into and out of the address space when processing data. Applications such as tilt-series tomography, spectrum-imaging and STEM diffraction imaging become slow and cumbersome as a result. Access to a much large address space has the potential to restore the speed of data manipulation in these applications.

In tomography a tilt series can be acquired covering the range -60 to 60 degrees with a 1 degree interval, or 120 images. If each image comes from a $4 \mathrm{k}$ camera it takes $32 \mathrm{MB}$ in storage (at 2 bytes per pixel). The complete dataset would be $3.8 \mathrm{~GB}$ in size. While it is possible to acquire this data by saving each acquired image to the hard drive, it would be hard or impossible to do the reconstruction of the complete dataset. The problem only gets worse if EFTEM Tomography is attempted, where multiple images are acquired at each tilt angle.

In spectrum-imaging the same problem arises when users try to acquire datasets consisting of more than 512 x 512 spectra. A spectrum image of that size, where each spectrum contains 2048 channels, at 2 bytes each, would require $1 \mathrm{~GB}$ of storage. With the introduction of $\mathrm{C}_{\mathrm{s}}$ probe-corrected STEMs, and the capability to acquire EELS spectra much faster with the GIF Quantum imaging filter, very large SI data sets can be acquired in a short period. To acquire the dataset described above would take only 4 minutes at 1000 spectra per second!

STEM diffraction imaging is a technique that enables diffraction patterns to be acquired over a grid of positions and stored in a 4D data-set. In this case relatively small scans result in extremely large data-sets: for example a grid of 128 x 128 pixels, with a 256 × 256 image recorded at each point would result in a $2 \mathrm{~GB}$ data-set.

In the last few years 64-bit versions of the Windows OS have become available. The price of RAM continues to decrease and computers with the x86-64 architecture, equipped with 8GB of RAM and running a 64-bit version of Windows have become quite affordable. A native 64-bit application running on such a system is currently limited to an address space of $16 \mathrm{~TB}$ ( $2^{44}$ bytes). In theory this limit can be raised in the future to $16 \mathrm{~EB}$ ( $2^{64}$ bytes) but it already overcomes the constraints imposed by the current 32-bit architecture and 4GB address limit.

With the release of GMS 2.0 Gatan is introducing a native 64-bit version of the industry standard DigitalMicrograph application. DigitalMicrograph-64 takes full advantage of the virtually unlimited memory space available, so users no longer have application induced restrictions on the size of their Tomography, SI or STEM DI datasets. Even though the main application is 64-bit, it is designed to still communicate with $3^{\text {rd }}$ party components that are only available in 32-bit format, such as 
microscopes, stages, and software libraries. This means that GMS 2.0 can run natively as 64-bit, but still interface with all of the microscopes supported in the past.

Other updates in GMS 2.0:

- Re-architected to use the latest user-interface paradigms popular on window applications, creating a fully customizable user experience.

- New FFT and DFT support using all available cores during processing. This results in processing times several times smaller than in earlier versions.

- The popular DM scripting language is supported using 64-bit assembly language for its inline processing.

- User specific preferences based on Windows user log-in.

- Support for Windows Vista and Windows 7.

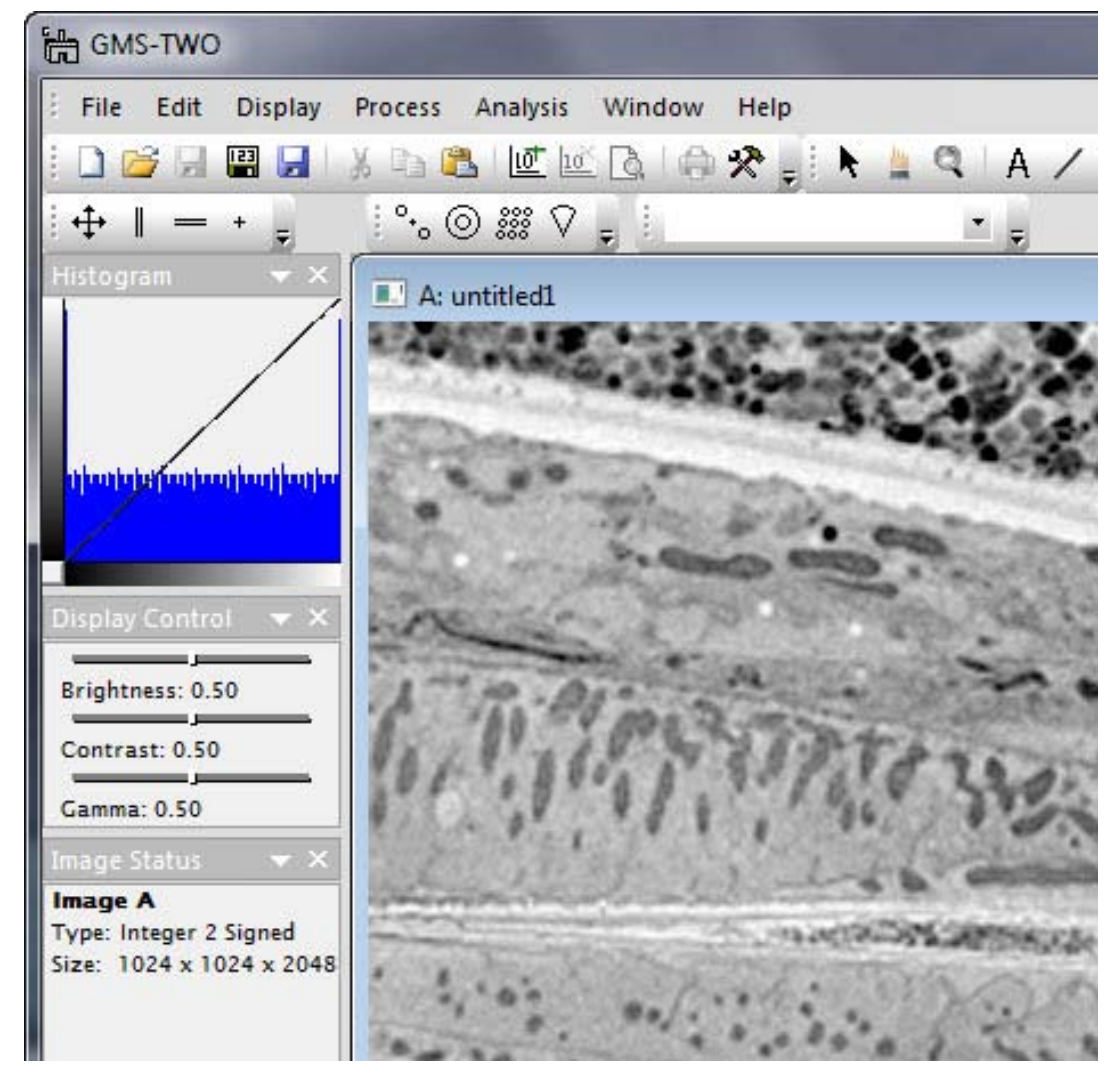

Fig. 1. Screenshot of GMS 2.0 (alpha) running as a 64-bit application - note the image size at 4GB. Also, the new dockable toolbars and floating windows can be seen. 